Bull. Fac.Agric.,Cairo Univ., 63: 152-161 (2012).

\title{
EFFECT OF ORGANIC AND CHEMICAL FERTILIZATION ON GROWTH, YIELD, AND ESSENTIAL OIL CONTENT OF FENNEL (Foeniculum vulgare Mill.)
}

( Received: 13.3.2012)

\author{
By \\ S. A. Al-Dalain \\ Al-Shoubak University College, Al-Balqa’ Applied University, Al-Salt 19117, Jordan.
}

\begin{abstract}
Two field experiments were carried out at a private farm at South Ghor area, Al-Karak, Jordan, during 2009/2010 and 2010/2011 cropping seasons. The study aimed to find the effect of four levels of organic fertilizer manure at $0,20,40$ and 60 ton/ha and three levels of chemical fertilizer at 0,30 , and $60 \mathrm{~kg} / \mathrm{ha}$ on growth, yield, and essential oil percentage of fennel (Foeniculum vulgare Mill.). The experimental design was complete randomized block design with split plot arrangement with three replications. The results showed that organic manure application combined with chemical fertilizers (NPK+ trace elements) significantly $(\mathrm{P}<0.01)$ increased plant height, number of branches, fresh and dry weights of herb, essential oil (\%) in fennel fruits, and essential oil yield/plant, as compared to pure chemical fertilizer treatments. Herb nitrogen and potassium contents were significantly $(\mathrm{P}<0.01)$ increased with organic fertilizer amendment or chemical fertilizer application, whereas herb-phosphorus content was not significantly affected by all fertilizer treatments. Soil electrical conductivity (EC) and organic matter content increased significantly by adding organic manure, while soil $\mathrm{pH}$ was not affected.
\end{abstract}

Key words: chemical fertilizer, essential oil, fennel, growth, organic manure, yield.

\section{INTRODUCTION}

Fennel plant (Foeniculum vulgare Mill.) a member of Apiaceae family, grown within the Mediterranean region, is a short- lived medicinal and aromatic herb. Its fruits are widely used in the preparation of various dishes like sauces, pickles, pastries, confectioneries, and soups (Bhati et al., 1988). It is also used in pharmaceutical and cosmetic products (Piccaglia and Marotti, 2001), in addition to its uses in the traditional Arabian medicine as diuretic and digestive appetizer (Karnick, 1994). Fennel fruits contain 1 to 3\% essential oils, which have disinfectant and antiinflammatory action on the respiratory and digestive organs and have an antispasmodic effect on smooth muscle (Stary and Jirasek, 1975). Leung and Foster (1996) reported that fennel oils are used to treat diseases like cholera bile disturbances, diarrhea, constipation, and nervous disorder. Recently, Anand et al., 2008 found that these oils possess anticancer activity.

Generally, excessive amounts of chemical fertilizers are applied to increase crops productivity (Nilsson,1979, Bengt and Martensoon, 2003). However, long-term applications of these fertilizers have caused a noticeable decrease in crop productivity (Chand $e t$ al., 2006), because it mainly contains major macro elements (NPK) in large quantities and are without the use of organic manures and caused deterioration of soil health by increasing soil salinization and in turn it affects human being and plants (Choudhry, 2005).

Organic fertilizers increase crop quality especially medicinal and aromatic plants, and it is more acceptable than chemical fertilizers (Abou El-Fadl et al., 1990), improve plant growth, yield, oil percentage (Ibrahim, 1999), and chemical constituents (Khalil, 2000), they have beneficial impacts on soil physical and chemical properties (Stevenson, 1994 ; O’Brien and Barker, 1996), result in better availability of soil micro-organisms (Zheljazkov and Warman, 2004), and provide energy for these microorganisms (Tejada et al., 2006)

Recently, consumers in Jordan are demanding higher quality and safer food especially organic products. In addition, fennel in Jordan is considered a promising medicinal and aromatic plant, that could be developed in Jordan for local and export purposes. The aim of this study was to determine the effects of different doses of organic 
and inorganic fertilizers on growth, yield and oil percentage of fennel.

\section{MATERIALS AND METHODS}

Two field experiments were carried out during the two winter cropping seasons of 2009/2010 and 2010/2011, at a private farm in South Ghor area, south Karak district, Jordan. Some physical and chemical characteristics of the experimental soil are shown in Table (1). Fertilizer treatments consisted of organic fertilizers (chicken, sheep \& cow manure at ratio1:1:1) with four levels of 0 , 20,40 and 60 ton/ha and N:P:K-chemical fertilizer (NPK 20-20-20+trace elements) at three levels of 0,30 and $60 \mathrm{~kg} / \mathrm{ha}$ monthly. Organic fertilizers were obtained from a Dairalla Company/Al-Salt for organic fertilizer production. Some chemical compositions of the organic manure are presented in Table (1). and contained 40 plants.

Chemical fertilizer (NPK 20-20-20+trace elements) was added monthly using a drip irrigation system. The first application was added 6 weeks after plantation then continued monthly in May. Irrigation commenced at the time of planting and continued throughout the growing season in both cropping seasons. Fennel was harvested at fruit maturity.

The following data were recorded: plant height (cm), number of branches/plant, fresh and dry weights (g/plant), fruit yield ( $\mathrm{g} /$ plant), essential oil (ml/plant). The essential oil from fennel fruits was obtained by hydro distillation for 3 hours in order to extract the essential oils using the method of Guenther (1961).

Concentrations of nitrogen, phosphorus, and potassium in the herb were determined using the method of (Cottenie et al., 1982). Soil samples

Table (1): Chemical analysis of the experimental soil and organic manure during two seasons.

\begin{tabular}{|c|c|c|c|}
\hline \multirow{2}{*}{ Chemical analysis } & \multicolumn{2}{|c|}{ Soil } & \multirow{2}{*}{ Organic manur } \\
\hline & $\begin{array}{c}\text { First season, } \\
2009 / 2010\end{array}$ & $\begin{array}{c}\text { Second season, } \\
2010 / 2011\end{array}$ & \\
\hline pH & 7.5 & 7.4 & 5.7 \\
\hline$E C\left(d S m^{-1}\right)$ & 2.32 & 2.24 & 3.63 \\
\hline $\operatorname{CaCO3}(\%)$ & 26 & 28 & 14.8 \\
\hline Organic matter(\%) & 2.23 & 2.19 & 24.66 \\
\hline Total N (\%) & 0.076 & 0.072 & 4.86 \\
\hline NaHCO3-P $\left(\mathrm{mg} \mathrm{kg}^{-1}\right)$ & 12 & 15 & nd* $^{*}$ \\
\hline Total Fe (ppm) & nd & nd & 1123 \\
\hline Total Zn (ppm) & nd & nd & 324 \\
\hline Total Mn (ppm) & nd & nd & 356 \\
\hline Available Fe (ppm) & 1.922 & 1.846 & nd \\
\hline Available Zn (ppm) & 1.876 & 1.578 & nd \\
\hline Available Mn (ppm) & 1.247 & 1.161 & nd \\
\hline
\end{tabular}

The soil was prepared for planting by plowing, disking and leveling. Organic fertilizer was incorporated into the soil of the respective plots to a depth of about $15 \mathrm{~cm}$ two weeks before transplantation, and saturated with irrigation water until it reaches its field capacity. Fennel seeds were sown in the two seasons on $7^{\text {th }}$ November. The experimental plot consisted of four rows, $3 \mathrm{~m}$ in length with $60 \mathrm{~cm}$ apart and a distance of $30 \mathrm{~cm}$ between plants within the row. Each plot consisted of four rows. The experimental design was a complete randomized block (CRBD) design with factorial arrangement of the treatments (Organic fertilizer and chemical fertilizer) with three replications. Each plot occupied an area of $7.2 \mathrm{~m}^{2}$ were taken from all plots at the end of the experiment for chemical analyses. These samples were dried, mixed thoroughly, pulverized in mortar and passed through a $2 \mathrm{~mm}$ sieve. Then samples were prepared for $\mathrm{pH}$, electric conductivity (EC) $(1: 2.5 \mathrm{w} / \mathrm{w})$ and organic matter (\%) determination according to Chapman and Pratt (1961).

Data were analyzed by two way analysis of variance using the SAS statistical package (SAS institute, 2003), and the differences between the means were compared using Fisher's least significant difference (LSD) at $\mathrm{P} \leq 0.05$ (Steel and Torrie., 1980). 


\section{RESULTS AND DISCUSSION \\ 3.1. Vegetative growth}

The results presented in Tables $(2 \& 3)$ show that the application of organic manure caused a huge increase in plant growth parameters, i.e. (plant height, number of branches/plant, fresh and dry weights of herbs) compared to the control and the chemical fertilizer treatments. The highest growth values were obtained at 40 or 60 tons organic manure fertilizer/ha. These results are in harmony with many other researchers who worked on different medicinal and aromatic crops. ElGhadban (1998) worked on Mentha spicate and Origanum majorana, Kandil (2002 ) on fennel (Foeniculum vulgare, Mill.); Naguib and Aziz (2003) on Hyosyamus muticus; and El-Sherbeny et al. (2005) on Sideritis montana L. All authors showed that vegetative growth of plants significantly increased with organic manure application. The improvement in yield might be due to the fact that organic fertilizers provide plants with different nutrients, and cause an improvement of soil water holding capacity as a result of organic manure application (Nilsson, 1979). According to Pimentel et al. (2005), organic manure increases the abundance of soil organisms by providing organic micronutrient for mycorrhiza, which encourage absorption of nutrients by plants. These nutrients became more available when soil $\mathrm{pH}$ decreases due to organic manure application( Salem., 1986 ).On the other hand, the combination of the high level of organic manure (40 or 60 ton/ha ) with (30 or $60 \mathrm{~kg} / \mathrm{ha}$ ) of chemical fertilizer resulted in significant increases in plant height, number of branches/plant, plant fresh and dry weights, compared with other combination treatments (Tables $2 \& 3$ ). Similar findings were obtained in Nigella sativa (Somida et al., 2001) and fennel plant (Badran and Safwat., 2004).

\subsection{Fruits and oil yield}

Tables $(4 \& 5)$ show the effect of different organic and inorganic (i. e. chemical fertilizers) on fruit yield (g/plant), oil yield ( $\mathrm{ml} / \mathrm{plant})$, and essential oil (\%). In general, adding chemical fertilizer in combination with organic fertilizer tended to increase fruit yield in comparison with the application of each of them alone.

The highest average fruit yield (76.7 g/plant) was obtained in 2009/2010 cropping season, while the average yield in 2010/2011 cropping season was $74.0 \mathrm{~g} / \mathrm{plant}$, obtained by adding 40 or 60 tons of organic fertilizer combined with 30 or $60 \mathrm{~kg} / \mathrm{ha}$ chemical fertilizer. The lowest fruit yield was obtained from untreated plot with a yield of 48.8 and $44.0 \mathrm{~g} / \mathrm{plant}$ in the first and second cropping seasons, respectively. Addition of 0 and $30 \mathrm{~kg} / \mathrm{ha}$ chemical fertilizer without organic fertilizer significantly $(\mathrm{P}<0.01)$ produced lower fruit yield compared with other treatment combinations. Essential oil (\%) in fennel fruits was significantly $(p<0.01)$ increased by all organic fertilizer levels when compared to the chemical fertilizer. The highest organic fertilizer level (40 or 60 ton/ha) alone or with chemical fertilizer treatments, resulted in a significant increment in oil (\%) compared with plots that untreated with organic fertilizer in both cropping seasons. It is clear that oil yield (ml /plant) had a parallel trend to oil (\%), the three organic fertilizer levels significantly raised oil yield over the chemical fertilizer treatments. Raising organic fertilizer level progressively increased fennel oil yield. It was clearly evident that all treatments that received organic fertilizer recorded higher fruits yield, oil yield as well as essential oil (\%), when compared with plots that did not receive organic fertilizer, indicating the importance of organic fertilizer in enhancing fennel productivity. The increase in the fruit yield, oil yield, and essential oil of fennel that resulted from organic fertilizer application might be due to the increase in soil organic content (Mona et al., 2008) and enhancement of soil fertility (Fortun et al., 1989), and stimulating effect of the organic manure that supplies plants with nutrient requirement for high yield and oil yield ( Hussein et al., 2006; Wong et al., 1999). The high fruit crop yield due to organic fertilizer could be attributed to favorable changes in soil condition which might result in loose soil and enables better root growth (Amanullah et al., 2006). In addition, the improvement of plant biomass is related to positive influence of organic fertilizer which causes slow and steady availability of nutrients throughout the growing season (Amanullah et al., 2006; Tejada et al., 2006).

\subsection{Chemical Composition}

Nitrogen content of fennel herb was significantly increased $(\mathrm{P}<0.01)$ by organic fertilizer application (Tables $6 \& 7$ ). The highest herb-N content (1.37 and $1.31 \%$ D.W in 2009/2010 and 2010/2011 cropping seasons, respectively) occurred in plots received 60 tons/ha organic fertilizer/ha) with $60 \mathrm{~kg}$ chemical fertilizer/ha, while the lowest herb-N content (1.04 and $0.96 \%$ D.W in 2009/2010 and 2010/2011 cropping seasons, respectively) was obtained from the plant in untreated plots. These results are in agreement with those obtained by 
Table (2): Interactive effect of organic manure and chemical fertilizer on plant height, number of branches, fresh weight and dry weight of fennel, Foeniculum vulgare Mill .during the two seasons.

\begin{tabular}{|c|c|c|c|c|c|c|c|c|c|}
\hline \multicolumn{2}{|c|}{ Fertilizer type } & \multicolumn{4}{|c|}{$\begin{array}{c}\text { First season, } \\
2009 / 2010\end{array}$} & \multicolumn{4}{|c|}{$\begin{array}{c}\text { Second season, } \\
2010 / 2011\end{array}$} \\
\hline $\begin{array}{l}\text { Organic } \\
\text { (ton/ha) }\end{array}$ & $\begin{array}{c}\text { Chemical } \\
\text { (kg/ha) }\end{array}$ & $\begin{array}{c}\text { Plant } \\
\text { height } \\
(\mathrm{cm})\end{array}$ & $\begin{array}{c}\text { Number } \\
\text { of } \\
\text { branches }\end{array}$ & $\begin{array}{c}\text { Plant } \\
\text { fresh } \\
\text { weight } \\
\text { (g) }\end{array}$ & $\begin{array}{l}\text { Plant dry } \\
\text { weight (g) }\end{array}$ & $\begin{array}{c}\text { Plant } \\
\text { height } \\
(\mathrm{cm})\end{array}$ & $\begin{array}{c}\text { Number } \\
\text { of } \\
\text { branches }\end{array}$ & $\begin{array}{c}\text { Plant } \\
\text { fresh } \\
\text { weight } \\
\text { (g) }\end{array}$ & $\begin{array}{c}\text { Plant } \\
\text { dry } \\
\text { weight } \\
\text { (g) }\end{array}$ \\
\hline \multirow{3}{*}{$\mathbf{0}$} & $\mathbf{0}$ & 135.9 & 4.8 & 364.6 & 87.9 & 126.5 & 4.5 & 329.0 & 80.9 \\
\hline & 30 & 136.0 & 5.1 & 373.8 & 93.2 & 126.8 & 4.7 & 336.5 & 83.7 \\
\hline & 60 & 137.1 & 5.3 & 380.1 & 96.3 & 127.2 & 5.1 & 345.1 & 86.9 \\
\hline \multirow{3}{*}{20} & $\mathbf{0}$ & 141.7 & 5.3 & 408.7 & 105.4 & 132.5 & 5.3 & 371.6 & 96.1 \\
\hline & 30 & 142.8 & 5.5 & 418.5 & 108.9 & 133.5 & 5.3 & 380.3 & 98.0 \\
\hline & 60 & 143.6 & 5.6 & 422.5 & 109.2 & 134.0 & 5.5 & 384.4 & 99.4 \\
\hline \multirow{3}{*}{40} & $\mathbf{0}$ & 143.1 & 5.7 & 460.0 & 121.5 & 135.1 & 5.5 & 420.6 & 102.3 \\
\hline & 30 & 144.3 & 5.7 & 466.2 & 123.7 & 135.9 & 5.6 & 431.7 & 113.6 \\
\hline & 60 & 144.3 & 5.9 & 470.5 & 125.3 & 136.1 & 5.7 & 434.8 & 114.3 \\
\hline \multirow{3}{*}{60} & $\mathbf{0}$ & 144.6 & 5.9 & 474.8 & 126.4 & 136.6 & 5.9 & 435.2 & 114.6 \\
\hline & 30 & 144.5 & 6.1 & 481.9 & 129.2 & 136.8 & 5.9 & 437.1 & 115.4 \\
\hline & 60 & 144.8 & 6.3 & 487.1 & 129.1 & 136.8 & 6.0 & 438.6 & 116.4 \\
\hline \multicolumn{2}{|c|}{$\operatorname{LSD}(0.05)$} & 0.96 & 0.19 & 3.32 & 2.17 & 0.63 & 0.16 & 3.54 & 2.98 \\
\hline
\end{tabular}

Table (3):Plant height, number of branches, fresh weight and dry weight of fennel, Foeniculum vulgare Mill as affected by organic manure and chemical fertilizer during the two seasons.

\begin{tabular}{|c|c|c|c|c|c|c|c|c|}
\hline \multirow[b]{2}{*}{ Treatments } & \multicolumn{4}{|c|}{$\begin{array}{c}\text { First season, } \\
2009 / 2010\end{array}$} & \multicolumn{4}{|c|}{$\begin{array}{c}\text { Second season, } \\
2010 / 2011\end{array}$} \\
\hline & $\begin{array}{c}\text { Plant } \\
\text { height } \\
(\mathrm{cm})\end{array}$ & $\begin{array}{c}\text { Number } \\
\text { of } \\
\text { branches }\end{array}$ & $\begin{array}{c}\text { Plant } \\
\text { fresh } \\
\text { weight } \\
\text { (g) }\end{array}$ & $\begin{array}{l}\text { Plant } \\
\text { dry } \\
\text { weight } \\
\text { (g) }\end{array}$ & $\begin{array}{c}\text { Plant } \\
\text { height } \\
(\mathrm{cm})\end{array}$ & $\begin{array}{c}\text { Number } \\
\text { of } \\
\text { branches }\end{array}$ & $\begin{array}{l}\text { Plant } \\
\text { fresh } \\
\text { weight } \\
\text { (g) }\end{array}$ & $\begin{array}{c}\text { Plant } \\
\text { dry } \\
\text { weight } \\
\text { (g) }\end{array}$ \\
\hline \multicolumn{9}{|l|}{$\begin{array}{c}\text { Organic } \\
\text { matter }\end{array}$} \\
\hline 0 & 136.27 & 5.02 & 371.83 & 92.45 & 125.83 & 4.71 & 336.87 & 83.84 \\
\hline 20 & 142.67 & 5.49 & 416.55 & 107.83 & 135.14 & 5.36 & 378.76 & 97.83 \\
\hline 40 & 143.57 & 5.76 & 465.56 & 123.53 & 135.29 & 5.62 & 429.07 & 116.23 \\
\hline 60 & 144.10 & 6.16 & 481.27 & 128.20 & 136.27 & 5.98 & 432.62 & 115.87 \\
\hline LSD(0.05) & 0.82 & 0.12 & 3.11 & 1.01 & 0.56 & 0.14 & 3.43 & 2.91 \\
\hline \multicolumn{9}{|l|}{$\begin{array}{l}\text { Chemical } \\
\text { fertilizer }\end{array}$} \\
\hline 0 & 136.22 & 5.40 & 426.24 & 110.30 & 132.99 & 5.28 & 387.84 & $\mathbf{1 0 2 . 8 9}$ \\
\hline 30 & 136.54 & 5.62 & 435.10 & 113.73 & 132.91 & 5.37 & 395.41 & 102.69 \\
\hline 60 & 137.37 & 5.80 & 440.10 & 114.98 & 133.49 & 5.60 & 399.73 & 102.75 \\
\hline LSD(0.05) & 0.71 & 0.10 & 2.70 & 0.87 & 0.49 & 0.12 & 2.97 & 2.52 \\
\hline Interaction & ns & $*$ & $*$ & $* *$ & $*$ & ns & ns & $*$ \\
\hline
\end{tabular}

$*$, ** and n.s. indicate $\mathbf{P}<0.05, P<0.01$ and not significant, respectively. 
Table (4): Interactive effect of organic manure and chemical fertilizer on fruit (yield $\mathrm{g}$ plant-1), essential oil (\%)and oil yield /plant(ml) of fennel, Foeniculum vulgare Mill. During the two cropping seasons.

\begin{tabular}{|c|c|c|c|c|c|c|c|}
\hline \multicolumn{2}{|c|}{ Fertilizer type } & \multicolumn{3}{|c|}{$\begin{array}{l}\text { First season, } \\
2009 / 2010\end{array}$} & \multicolumn{3}{|c|}{$\begin{array}{c}\text { Second season, } \\
2010 / 2011\end{array}$} \\
\hline $\begin{array}{l}\text { Organic } \\
\text { (ton/ha) }\end{array}$ & $\begin{array}{l}\text { Chemica } \\
\text { l (kg/ha) }\end{array}$ & $\begin{array}{c}\text { Fruit } \\
\text { (yield g } \\
\text { plant-1) }\end{array}$ & $\begin{array}{l}\text { Oil } \\
(\%)\end{array}$ & $\begin{array}{c}\text { Oil yield } \\
\text { /plant(ml) }\end{array}$ & $\begin{array}{c}\text { Fruit } \\
\text { (yield g } \\
\text { plant-1) }\end{array}$ & $\begin{array}{l}\text { Oil } \\
(\%)\end{array}$ & $\begin{array}{c}\text { Oil yield } \\
\text { /plant }(\mathbf{m l})\end{array}$ \\
\hline \multirow[b]{3}{*}{$\mathbf{0}$} & $\mathbf{0}$ & 48.8 & 1.86 & 0.91 & 44.0 & 1.84 & 0.81 \\
\hline & 30 & 51.2 & 2.30 & 1.18 & 46.1 & 2.30 & 1.06 \\
\hline & 60 & 53.1 & 2.31 & 1.23 & 51.1 & 2.32 & 1.18 \\
\hline \multirow{3}{*}{20} & $\mathbf{0}$ & 57.9 & 2.50 & 1.45 & 55.0 & 2.37 & 1.30 \\
\hline & 30 & 61.8 & 2.52 & 1.56 & $\begin{array}{l}57.8 \\
\end{array}$ & 2.38 & 1.38 \\
\hline & 60 & 63.4 & 2.52 & 1.60 & 60.0 & 2.39 & 1.43 \\
\hline \multirow[b]{3}{*}{40} & $\mathbf{0}$ & 74.3 & 2.55 & 1.89 & 70.2 & 2.40 & 1.68 \\
\hline & 30 & 75.5 & 2.57 & 1.94 & 73.0 & 2.40 & 1.75 \\
\hline & 60 & 75.4 & 2.57 & 1.94 & 73.2 & 2.41 & 1.76 \\
\hline \multirow[b]{3}{*}{60} & 0 & 75.6 & 2.55 & 1.93 & 73.4 & 2.40 & 1.76 \\
\hline & 30 & 76.7 & 2.56 & 1.96 & 73.8 & 2.42 & 1.79 \\
\hline & 60 & 76.1 & 2.57 & 1.96 & 74.0 & 2.43 & 1.80 \\
\hline \multicolumn{2}{|l|}{$\operatorname{LSD}(0.05)$} & 1.23 & 0.08 & 0.05 & 1.21 & 0.08 & 0.06 \\
\hline
\end{tabular}

Table (5) : Fruit (yield g plant-1), essential oil (\%), and oil yield/ plant (ml) of fennel, Foeniculum vulgare Mill as affected by organic manure and chemical fertilizer during the two cropping seasons.

\begin{tabular}{|c|c|c|c|c|c|c|}
\hline \multirow[t]{2}{*}{ Treatments } & \multicolumn{3}{|c|}{$\begin{array}{l}\text { First season, } \\
2009 / 2010\end{array}$} & \multicolumn{3}{|c|}{$\begin{array}{c}\text { Second season, } \\
2010 / 2011\end{array}$} \\
\hline & $\begin{array}{l}\text { Fruit (yield } \\
\text { g plant-1) }\end{array}$ & $\begin{array}{l}\text { Oil } \\
(\%)\end{array}$ & $\begin{array}{l}\text { Oil yield } \\
\text { /plant } \\
\text { (ml) }\end{array}$ & $\begin{array}{l}\text { Fruit (yield } \\
\text { g plant-1) }\end{array}$ & $\begin{array}{l}\text { Oil } \\
(\%)\end{array}$ & $\begin{array}{l}\text { Oil yield } \\
\text { /plant } \\
\text { (ml) }\end{array}$ \\
\hline \multicolumn{7}{|l|}{$\begin{array}{l}\text { Organic } \\
\text { matter }\end{array}$} \\
\hline $\mathbf{0}$ & 51.02 & 1.88 & 0.96 & 47.09 & 1.85 & 0.87 \\
\hline 20 & 61.01 & 2.42 & 1.48 & 57.59 & 2.26 & 1.30 \\
\hline 40 & 75.07 & 2.53 & 1.90 & 71.75 & 2.37 & 1.70 \\
\hline 60 & 76.13 & 2.56 & 1.95 & 71.68 & 2.38 & 1.71 \\
\hline $\operatorname{LSD}(0.05)$ & 1.10 & 0.14 & 0.07 & 0.94 & 0.15 & 0.028 \\
\hline \multicolumn{7}{|l|}{$\begin{array}{l}\text { Chemical } \\
\text { fertilizer }\end{array}$} \\
\hline $\mathbf{0}$ & 50.83 & 1.91 & 0.97 & 45.54 & 1.84 & 0.84 \\
\hline 30 & 54.30 & 2.27 & 1.23 & 48.29 & 2.22 & 1.07 \\
\hline 60 & 55.72 & 2.33 & 1.30 & 52.15 & 2.23 & 1.22 \\
\hline $\operatorname{LSD}(0.05)$ & 0.96 & 0.11 & 0.06 & 0.81 & 0.18 & 0.012 \\
\hline Interaction & $*$ & $* *$ & $*$ & $*$ & $*$ & $*$ \\
\hline
\end{tabular}

*, ** and $\mathrm{n}$. .s. indicate $\mathrm{P}<0.05, \mathrm{P}<0.01$ and not significant, respectively. 
Table (6): Interactive effect of organic manure and chemical fertilizer on chemical composition of fennel, Foeniculum vulgare Mill. during the two seasons.

\begin{tabular}{|c|c|c|c|c|c|c|c|}
\hline \multicolumn{2}{|c|}{ Fertilizer type } & \multicolumn{3}{|c|}{$\begin{array}{c}\text { First season, } \\
2009 / 2010\end{array}$} & \multicolumn{3}{|c|}{$\begin{array}{c}\text { Second season, } \\
2010 / 2011\end{array}$} \\
\hline $\begin{array}{r}\text { Organic } \\
\text { (ton/ha) }\end{array}$ & $\begin{array}{c}\text { Chemical } \\
\text { (kg/ha) }\end{array}$ & N \% & P \% & K \% & N\% & P \% & K \% \\
\hline \multirow{3}{*}{$\mathbf{0}$} & $\mathbf{0}$ & 1.04 & 0.21 & 2.55 & 0.96 & 0.20 & 2.53 \\
\hline & 30 & 1.14 & 0.25 & 3.38 & 1.07 & 0.24 & 3.37 \\
\hline & 60 & 1.18 & 0.26 & 3.42 & 1.12 & 0.26 & 3.43 \\
\hline \multirow{3}{*}{20} & $\mathbf{0}$ & 1.12 & 0.24 & 2.93 & 1.07 & 0.25 & 2.90 \\
\hline & 30 & 1.16 & 0.26 & 3.50 & 1.12 & 0.26 & 3.47 \\
\hline & 60 & 1.21 & 0.27 & 3.52 & 1.16 & 0.26 & 3.50 \\
\hline \multirow{3}{*}{40} & $\mathbf{0}$ & 1.16 & 0.26 & 3.07 & 1.12 & 0.25 & 3.04 \\
\hline & 30 & 1.23 & 0.27 & 3.52 & 1.17 & 0.25 & 3.51 \\
\hline & 60 & 1.30 & 0.27 & 3.54 & 1.26 & 0.26 & 3.52 \\
\hline \multirow{3}{*}{60} & $\mathbf{0}$ & 1.24 & 0.25 & 3.23 & 1.25 & 0.25 & 3.19 \\
\hline & 30 & 1.31 & 0.27 & 3.56 & 1.27 & 0.26 & 3.50 \\
\hline & 60 & 1.37 & 0.26 & 3.51 & 1.31 & 0.26 & 3.54 \\
\hline \multicolumn{2}{|c|}{ LSD(0.05) } & 0.03 & ns & 0.09 & 0.03 & 0.05 & 0.11 \\
\hline
\end{tabular}

Table (7): Chemical composition of fennel, Foeniculum vulgare Mill as affected by organic manure and chemical fertilizer during the two cropping seasons.

\begin{tabular}{|c|c|c|c|c|c|c|}
\hline \multirow[t]{2}{*}{ Treatments } & \multicolumn{3}{|c|}{$\begin{array}{l}\text { First season, } \\
2009 / 2010\end{array}$} & \multicolumn{3}{|c|}{$\begin{array}{c}\text { Second season, } \\
2010 / 2011\end{array}$} \\
\hline & $\mathbf{N} \%$ & P \% & K \% & $\mathbf{N} \%$ & P \% & K \% \\
\hline \multicolumn{7}{|c|}{ Organic matter } \\
\hline $\mathbf{0}$ & 1.05 & 0.24 & 2.58 & 0.97 & 0.24 & 2.54 \\
\hline 20 & 1.16 & 0.26 & 2.96 & 1.09 & 0.26 & 2.92 \\
\hline 40 & 1.23 & 0.27 & 3.10 & 1.14 & 0.26 & 3.06 \\
\hline 60 & 1.31 & 0.26 & 3.24 & 1.26 & 0.26 & 3.21 \\
\hline LSD(0.05) & 0.02 & ns & 0.07 & 0.02 & ns & 0.06 \\
\hline \multicolumn{7}{|c|}{ Chemical fertilizer } \\
\hline $\mathbf{0}$ & 1.06 & 0.22 & 2.57 & 0.98 & 0.21 & 2.55 \\
\hline 30 & 1.16 & 0.26 & 3.40 & 1.09 & 0.23 & 3.39 \\
\hline 60 & 1.19 & 0.27 & 3.45 & 1.14 & 0.26 & 3.48 \\
\hline $\operatorname{LSD}(0.05)$ & $\mathbf{0 . 0 3}$ & ns & 0.06 & 0.02 & 0.05 & 0.05 \\
\hline Interaction & $* *$ & ns & $*$ & $*$ & ns & $*$ \\
\hline
\end{tabular}

*, ** and n.s. indicate $P<0.05, P<0.01$ and not significant, respectively. 
Table (8): Interactive effect of organic manure and chemical fertilizer on soil pH, EC and organic matter content of fennel, Foeniculum vulgare Mill. during the two seasons.

\begin{tabular}{|c|c|c|c|c|c|c|c|}
\hline \multicolumn{2}{|c|}{ Fertilizer type } & \multicolumn{3}{|c|}{$\begin{array}{c}\text { First season, } \\
2009 / 2010\end{array}$} & \multicolumn{3}{|c|}{$\begin{array}{c}\text { Second season, } \\
2010 / 2011\end{array}$} \\
\hline $\begin{array}{l}\text { Organic } \\
\text { (ton/ha) }\end{array}$ & $\begin{array}{c}\text { Chemical } \\
\text { (kg/ha) }\end{array}$ & pH & $\begin{array}{c}\mathrm{EC} \\
\left(\mathrm{dS} \mathrm{m^{-1 }}\right)\end{array}$ & $\begin{array}{l}\text { OM } \\
(\%)\end{array}$ & $\mathbf{p H}$ & $\begin{array}{c}\mathrm{EC} \\
\left(\mathrm{dS} \mathrm{m}^{-1}\right)\end{array}$ & $\begin{array}{l}\mathrm{OM} \\
(\%)\end{array}$ \\
\hline \multirow{3}{*}{$\mathbf{0}$} & $\mathbf{0}$ & 7.4 & 2.34 & 2.55 & 7.4 & 2.24 & 2.48 \\
\hline & 30 & 7.5 & 2.33 & 2.59 & 7.5 & 2.24 & 2.52 \\
\hline & 60 & 7.6 & 2.33 & 2.57 & 7.5 & 2.24 & 2.49 \\
\hline \multirow{3}{*}{20} & $\mathbf{0}$ & 7.3 & 2.39 & 2.78 & 7.4 & 2.31 & 2.69 \\
\hline & 30 & 7.4 & 2.40 & 2.75 & 7.4 & 2.30 & 2.73 \\
\hline & 60 & 7.4 & 2.39 & 2.80 & 7.4 & 2.28 & 2.66 \\
\hline \multirow{3}{*}{40} & $\mathbf{0}$ & 7.4 & 2.46 & 3.04 & 7.3 & 2.38 & 2.98 \\
\hline & 30 & 7.3 & 2.47 & 3.02 & 7.4 & 2.37 & 2.94 \\
\hline & 60 & 7.4 & 2.45 & 3.07 & 7.4 & 2.35 & 2.89 \\
\hline \multirow{3}{*}{60} & $\mathbf{0}$ & 7.3 & 2.55 & 3.17 & 7.3 & 2.47 & 3.08 \\
\hline & 30 & 7.3 & 2.53 & 3.21 & 7.3 & 2.45 & 3.11 \\
\hline & 60 & 7.4 & 2.52 & 3.19 & 7.3 & 2.45 & 3.06 \\
\hline & LSD(0.05) & Ns & 0.08 & 0.08 & ns & 0.09 & 0.07 \\
\hline
\end{tabular}

n.s. indicate not significant

Table (9): Soil pH, EC and organic matter content of fennel, Foeniculum Vulgare Mill as affected by organic manure and chemical fertilizer during the two seasons.

\begin{tabular}{|c|c|c|c|c|c|c|}
\hline \multirow[t]{2}{*}{ Treatments } & \multicolumn{3}{|c|}{$\begin{array}{c}\text { First season, } \\
2009 / 2010\end{array}$} & \multicolumn{3}{|c|}{$\begin{array}{c}\text { Second season, } \\
2010 / 2011\end{array}$} \\
\hline & pH & $\begin{array}{c}E C \\
\left(\mathrm{dS} \mathrm{m}^{-1}\right)\end{array}$ & $\begin{array}{l}\text { OM } \\
(\%)\end{array}$ & pH & $\begin{array}{c}E C \\
\left(d S ~ m^{-1}\right)\end{array}$ & $\begin{array}{l}\text { OM } \\
(\%)\end{array}$ \\
\hline \multicolumn{7}{|l|}{$\begin{array}{l}\text { Organic } \\
\text { matter }\end{array}$} \\
\hline 0 & 7.4 & 2.32 & 2.55 & 7.4 & 2.25 & 2.50 \\
\hline 20 & 7.4 & 2.37 & 2.82 & 7.4 & 2.33 & 2.70 \\
\hline 40 & 7.4 & 2.46 & 3.08 & 7.3 & 2.38 & 2.97 \\
\hline 60 & 7.3 & 2.56 & 3.19 & 7.3 & 2.48 & 3.14 \\
\hline LSD(0.05) & ns & 0.07 & 0.04 & ns & 0.08 & 0.06 \\
\hline \multicolumn{7}{|l|}{$\begin{array}{l}\text { Chemical } \\
\text { fertilizer }\end{array}$} \\
\hline 0 & 7.4 & 2.34 & 2.58 & 7.4 & 2.25 & 2.41 \\
\hline 30 & 7.5 & 2.36 & 2.56 & 7.6 & 2.27 & 2.57 \\
\hline 60 & 7.5 & 2.36 & 2.63 & 7.5 & 2.28 & 2.55 \\
\hline LSD(0.05) & ns & 0.01 & 0.02 & ns & 0.01 & 0.03 \\
\hline Interaction & ns & $*$ & $* *$ & ns & $* *$ & $* *$ \\
\hline
\end{tabular}

*, ** and n.s. indicate $\mathbf{P}<0.05, P<0.01$ and not significant, respectively. 
Yuonis et al. (2004) on Ammi visnaga, L and Kandeel and Sharaf (2003) on marjoram plant. Tejada et al. (2006) attributed this increase in nutrient concentration in plants amended with organic manure to the increase in quantity and activity of soil microorganisms, which in turn resulted in a considerable accumulation of $\mathrm{N}$ in plants. Moreover, Goyal et al. (1993) reported that the incorporation of organic amendments to soil profile increases enzymatic activities. Organic matter contains intra- and extra cellular enzymes and also it might stimulate microbial activity in the soil.

The obtained results on herb $-\mathrm{P}$ and $\mathrm{K}$ content (\%) are presented (Tables, $6 \& 7$ ). herb -P content was not significantly affected by all fertilizer treatments in both cropping seasons. However, herb $-\mathrm{K}$ content was positively affected by all fertilizer treatments in comparison with untreated plots in both cropping seasons. These results are in line with those obtained by Al-Fraihat et al. (2011) who reported that the increment in $\mathrm{K}$ content of marjoram plant may explain the efficiency of suitable quantity of organic fertilizers that can attract and hold more nutrients and water on its surface to supply the plants with suitable amounts for a long time.

The application of organic manure either alone or in combination with NPK positively affected $\mathrm{N}, \mathrm{P}$ and $\mathrm{K}$ contents of fennel plants. The promotive effect of this character by low compost and mineral level may be due to the important role of $\mathrm{N}$ in metabolic processes like photosynthesis and carbohydrate synthesis(Naguib and Aziz ., 2003).

\subsection{Certain soil characteristics}

The data presented in (Tables 8 \& 9) show some soil chemical analysis at the end of the experiment. The results revealed that soil $\mathrm{pH}$ was not significantly affected by adding organic and chemical fertilizers or both. This could be due to the high soil carbonate content that can fix any change in its $\mathrm{pH}$ during organic matter decomposition. Similar results for soil $\mathrm{pH}$ were obtained by Abu-Zahra and Tahboub (2008). Soil E.C was significantly $(\mathrm{P}<0.01)$ increased by adding the chemical fertilizer with organic manure compared with those received chemical fertilizer. This could be attributed to the addition of organic fertilizers that supplied soil with soluble compounds (Wong et al., 1999).

Application of different doses of organic manure with or without chemical fertilizer significantly $(\mathrm{P}<0.01)$ enhanced soil organic matter content, however, the application of chemical fertilizer alone had no effects on soil organic matter content in both cropping seasons. These results are in harmony with those obtained by Herencia et al. (2007) and Theodora et al. (2003).

In conclusion fennel plants can be grown better in soil amended with organic manure as a fertilizer but application rate and availability of all minerals should be considered. Fennel plants grown in soil amended with organic manure showed a vigorous vegetative growth (plant height, number of branches, fresh and dry weights), high fruit and oil yield compared with plots received only chemical fertilizer. Further studies are needed to determine optimal rates of fertilizers to be used for proper growth and production of fennel crop.

\section{REFERENCES}

Abou El-Fadl I. A., Abou-Baker M. and ElGamal A. M. (1990). Effect of different organic manure composts on roselle (Hibiscus sabdariffa L.) plants and soil characteristics. Agic. Res. Review, 68 (5):1077-1087.

Abu-Zahra T.R. and Tahboub A. B. (2008). Effect of organic matter sources on chemical properties of the soil and yield of strawberry under organic farming conditions. World Appl. Sci. J., 5 (3): 383388.

Al-Fraihat A.H., Al-DalainS Y., Ziad B. AlRawashdeh Z.B., Mohammad S., Abu Darwish M.S. and Al-Tabbal J.A. (2011). Effect of organic and biofertilizers on growth, herb yield and volatile oil of marjoram plant grown in Ajloun region, Jordan. J. Med. Plant. Res. 5(13):28222833.

Amanullah M. M., Vaiyapuri K. and Alagesan A. (2006). Effect of intercropping and organic manures on the yield and biological efficiency of cassava intercropping system (Manihot esculenta Crantz). Res. J. Agric. Biol. Sci., 2(5): 201-208.

Anand P., Kunnumakara A., Sundaram C., Harikumar K., Tharakan S., Lai O., Sung B. and Aggarwal B. (2008). Cancer is a preventable disease that requires major lifestyle changes. Pharmaceut. Res., 25, 2097-2116.

Badran F.S. and Safwat M.S. (2004). Response of fennel plants to organic manure and bio-fertilizers in replacement of chemical fertilization. Egypt. J. Agric. Res., 82(2): 247- 256. 
Bengt L. and Martensson A. (2003). Organically produced plant food evidence of health benefits. Soil and Plant Sci., 53: 315.

Bhati D. S., Shaktawat M. S.., Somani L. L. and Agarwal H. R. (1988). Response of fennel (Foeniculum vulgare Mill.) to nitrogen and phosphorus. Transactions of Indian Society of Desert Technology, No. 2; 79-83.

Chand S., Anwar M. and Patra D.D. (2006). Influence of long-term application of organic and inorganic fertilizer to build up soil fertility and nutrient uptake in mintmustard cropping sequence. Communications in Soil Science and Plant Analysis. 37: 63-76.

Chapman H. D. and Pratt P. F. (1961). Methods of Analysis for Soils, Plant and Water. Univ. of California, USA. Translated to Arabic, 1996, Omar Mokhtar University, Libya.

Choudhry A.U. (2005). Higher-Value Organics, pp: 35-8. Pakistan and Gulf Economist.

Cottenie A., Verloo M., Velghe M. and Camerlynck R. (1982). Chemical Analysis of Plant and Soil. Manual Laboratory of Analytical and Agro chemistry. Ghent State Univ. Press, Belgium.

El-Ghadban E.A.E. (1998). Effect of some organic and inorganic fertilizers on growth, oil yield and chemical composition of spearmint and marjoram plants. Ph.D. Thesis, Fac. Agric.,Cairo Univ., Egypt .

El-Sherbeny S. E., Khalil M.Y. and Naguib N.Y. (2005). Influence of compost levels and suitable spacing on the productivity of Sideritis montana L. plants recently cultivated under Egyptian conditions. Bull. Fac. Agric., Cairo Univ.,56(2):373-392.

Fortun C., Fortun A. and Almendros G. (1989). The effect of organic materials and their humified fractions on the formation and stabilization of soil aggregates. The Science of the Total Environment, 81/82: 561-568.

Goyal S., Mishra M. M., Dhankar S. S., Kapoor K.K. and Batra R. (1993). Microbial biomass turnover and enzyme activities following the application of farmyard manure to field soils with and without previous long-term applications. Biol. Fertile. Soils, 15: 60-64.

Guenther E., (1961). The Essential Oils. D. von Nostrand Comp. Press, New York.
Herencia J. F., Ruiz-Porras J. C., Melero S., Garcia- Galavis P. A., Morillo E. and Maqueda C.( 2007). Comparison between organic and mineral fertilization for soil fertility levels, crop macronutrient concentrations and yield. Agron. J., 99: 973-983.

Hussein M. S., El-Sherbeny S. E., Khalil M.Y., Naguib N.Y. and Aly S. M. (2006). Growth characters and chemical constituents of Dracocephalum moldavica L. plants in relation to compost fertilizer and planting distance. Scientica Horticulturae, 108(3): 322- 331.

Ibrahim M. E., (1999). Physiological and chemical studies on tusli plant (Ocimum sanctum). Egypt. J. Hort., 26(2): 147-165.

Kandeel A.M. and Sharaf M.S. (2003). Productivity of Majorana hortensis, L. plants as influenced by the interaction between mineral and biological fertilization. J. Agric. Sci. Mansoura Univ., 28: 13731389.

Kandil M.A.M. (2002). The effect of fertilizers for conventioal and organic farmingon yield and oil quality of fennel (Foeniculum vulgare Mill.) in Egypt. Ph.D.Thesis. Braunschweing: FAl,94p, Landbanforsch Volkenrode SH 237, isbn 3-933140-60-9.

Karnick C. R. (1994). Pharmacopoeial standards of herbal plants, Vol. 1-2. Delhi: Sri. Atguru Publications. Vol. 1: 139-141; Vol. 2:71.

Khalil M.Y., (2000). Influence of compost and foliar fertilization on growth and chemical composition of Rosmarinus officinalis. Egypt. J. Appl. Sci., 17: 684-699.

Leung A.Y. and Foster S. (1996). Encyclopedia of Common Natural Ingredients Used in Food, Drugs, and Cosmetics, $2^{\text {nd }}$ ed. New York: John Wiley \& Sons, Inc.

Mona Y., Khalil M.A., Kandil M., and Swaefy Hend M.F. (2008). Effect of three different compost levels on fennel and salvia growth character and their essential oils. Res. J. Agric. \& Biol. Sci., 4(1): 34-39.

Naguib N.Y. and Aziz E.E. (2003). Yield and quality of Hyoscyamus muticus L.in relation to some fertilizer treatments. Egyptian Jour. of Hort., 30(1\2): 1-17.

Nilsson T. (1979). Yield, storage ability, quality and chemical composition of carrot, cabbage and leek at conventional and organic fertilizing. Acta Hort., 93: 209-223. 
O’Brien T.A. and Barker A.V. (1996). Growth of peppermint in several types of composts. J. Herbs, Spices \& Medicin. Plants, 4(1): 1927.

Piccaglia R. and Marotti M. (2001). Characterization of some Italian types of wild fennel (Foeniculum vulgare Mill.) J. Agric. Food Chem., 49, 239-244.

Pimentel D., Paul H., James H., David D. and Rita S. (2005). "Environmental, energetic, and economic comparisons of organic and conventional farming systems". BioScience., 55(7): 573-582.

Salem N. M. M. (1986). Agro-chemical aspects related to the use of conditioners and organic wastes in soils. Ph. D. Thesis, Fac. of Agric. Sci. Rijksuniv. Gent., Belgium.

Somida E. G., Attia F. A., Badran F. S. and ElSayed A.A. (2001). Response of Nigella sativa, L. plant, grown in sandy soil, to some nitrogen and potassium fertilization treatments. Proc. 5 Arabian Hort. Conf., th Ismailia, Egypt, March, 24-28: 179-194.

Stary F. and Jirasck V. (1975). A Concise Guide in Colour Herbs. Hamlyn; London, New York, Sydney, Toronto.

Steel R.G.D. and Torrie J.H. (1980). Principles and procedures of statistics. Published by Mcgraw-Hill Book Company, Inc. New
York. 1960.

Stevenson F.J. (1994). Humus Chemistry: Genesis, Composition Reaction. 2 Ed. John Wiley and Sons, Inc., New York.

Tejada M., Hernandez M. T. and Garcia C (2006). Application of two organic amendments on soil restoration: Effects on the soil biological properties. J. Environ. Qual., 35: 1010-1017.

Theodora M., Anastasios S.L. and Athanasios A.G. (2003). Effects of injected liquid cattle manure on growth and yield of winter wheat and soil characteristics. Agron. J., 95: 592-596

Wong J. W. C., Ma K. K., Fang K. M. and Cheung C. (1999). Utilization of manure compost for organic farming in Hong Kong. Bioresource Technology, 67: 43-46.

Yuonis S.I., Ghaly N.G. and Ahmed S. K. (2004). Effect of FYM and planting space on the vegetative growth, active ingredient and chemical composition of Ammi visnaga, $\mathrm{L}$. J. Agric. Sci. Mansoura Univ., 29(4): 19851993.

Zheljazkov V.D. and Warman P. R. (2004). Application of high $\mathrm{Cu}$ compost to dill and peppermint. J. Agric. Food Chem., 52: 2615-622.

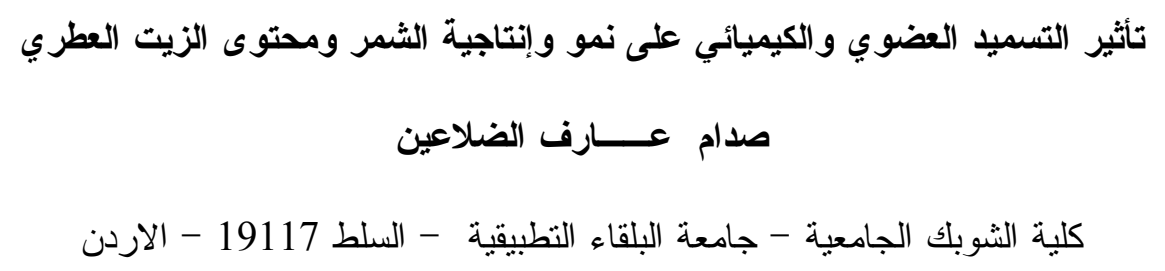

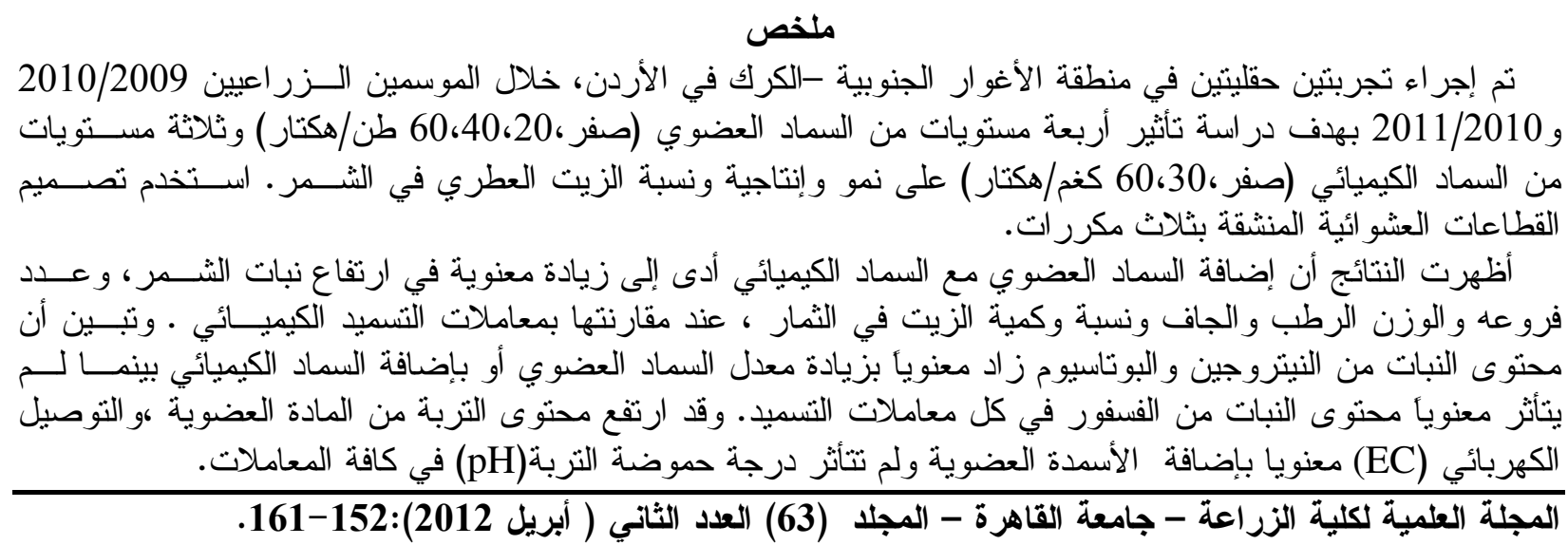

